\title{
Genetic code as an image of the mirror image. Part II
}

\author{
Miloje M. Rakočević \\ University of Niš, Faculty of Sciences and Mathematics, Department of Chemistry, \\ Višegradska 33, 18000 Niš, Serbia \\ The eternal mystery of the world is its comprehensibility... \\ The fact that it is comprehensible is a miracle.
}

Albert Einstein

\begin{abstract}
This unifying paper represents a compilation of three small works, published in OSF Preprints. All three works are related to the first part of the paper entitled with the same title as this, they are practically its continuation. The reason for giving up writing a special text as "Part II" and declaring two supplements and one scientific note as "Part II" lies in the epilogue of the first part of this paper, which (epilogue) I give here in Appendix.
\end{abstract}

Keywords: Protein amino acids, Amino acid code, Genetic code, Binary tree, Gray code, Golden mean, Fibonacci series, Periodic system, Mirror image, Mirroring.

PART I

GC_as_an_image

Created: November 19, 2019

\section{Preprint DOI}

\subsection{9/osf.io/xht4c}

SUPPLEMENT 1

GC as image. Suppl. 1

Created: December 30, 2019

\section{Preprint DOI}

10.31219/osf.io/umnt3
SYSTEM CHEMSTRY

System Chemistry

Created: February 13, 2020

Preprint DOI

10.31219/osf.io/z7bm3

SUPPLEMENT 2

GC as image. Suppl. 2

Created: March 31, 2020

Preprint DOI

10.31219/osf.io/6hbdn

Miloje M. Rakočević www.rakocevcode.rs mirkovmiloje@gmail.com 


\title{
Genetic code as an image of the mirror image. Supplement 1
}

\author{
Miloje M. Rakočević \\ University of Niš, Faculty of Sciences and Mathematics, Department of Chemistry, \\ Višegradska 33, 18000 Niš, Serbia
}

\begin{abstract}
Searching for the answer to the question why - in the generating of the genetic code - only mirror symmetrical left and not right amino acids (AAs) were selected, in a previous work we showed the existence of a double Boolean "triangle" in mirror symmetry, with superposition of the top vertices: 00 -11-22 / 22-11-00 $\rightarrow$ 00-11-22-11-00 [0 as 000; 1 as 001; 2 as 010] (Rakočević, 2019a). The resulting sequence, summed with the binary sequence of a 6-bit binary tree, split with a mirror in the middle (101/010) [as in Dirac's positron / electron mirror], results in a sequence of decimal number system: 02-13-24-16-05, where a smaller number $(010=2)$ was added three times and a larger number $(101=5)$ twice (Survey 1$)$. The mirror image of the obtained decimal sequence (20-31-42-61-50) is 100\% consistent with the arrangement of protein AAs, arranged according to strict chemical similarity (Rakočević, 2019a, Table 3). Starting from this result, the paper of which this is a supplement, presents new insights and new examples of mirror symmetry valid for the genetic code, showing that mirror symmetry is also in other respects an essential feature of the genetic code. In this Supplement are given the further new insights.
\end{abstract}

Keywords: Protein amino acids, Genetic code, Binary tree, Golden mean, Periodic system, Mirror image. Mirroring.

With all that said in the original paper on "Genetic code as an image of the mirror image" (Rakočević, 2019b) ${ }^{1}$ of which this text is a Supplement, here are given some additional new insights into the genetic code (GC). First, we show that it makes sense to test the existence of balancing and nuancing ${ }^{2}$ of the number of atoms in amino acid molecules, correspondent with their (molecular) physicochemical properties in $\mathrm{PAAS}^{3}$ in a new way. First, with the distinction through the first four AAs, which are the first and in their stereochemical types: Gly, the first, i.e. the simplest (and only) in the glycine type;

\footnotetext{
${ }^{1}$ It is understood that the reader is also familiar with the previous article "Protein amino acids as a complete (periodic) system" (Rakočević, 2019a).

${ }^{2}$ The notions of balancing and nuancing related to the state of the genetic code, as well as the conditions of

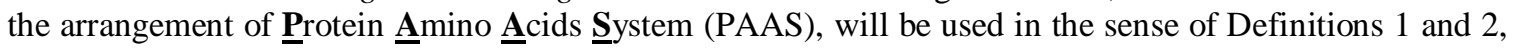
given in our previous paper (Rakočević, 2018a, p. 33).

${ }^{3}$ Rakočević, 2019a: "... the most complete Protein Amino Acids System (PAAS). It is shown that 20 protein amino acids appear to be a complete system - ordered, coherent, and harmonic. In such a system, all chemical distinctions within the system are accompanied by specific arithmetical and algebraic regularities, including the existence of amino acid ordinal numbers from 1 to 20 . The classification of amino acids into two decades (1-10 and 11-20) appears to be in a strict correspondence with the balances of the number of atoms" (Rakočević, 2019a, Table 2, p. 14).
} 
Ala, the first, i.e. the simplest in the set of 16 AAs of alanine stereochemical type; Val, the first, i.e. the simplest in the set of two AAs of valine stereochemical type; Pro, the first, i.e. the simplest (and only) in the proline type. ${ }^{4}$ The result given in Table 1 is proof of the affirmation of this query. The next symmetric and chemically meaningful, and questionable, distinction is the division into $(5+5)$ and $(5+5)$ of AAs. The result given in Table 2 is proof of the affirmation of this query. ${ }^{5}$

[Notice the validity of the principle of self-similarity in the relation between the arrangement given in Table 2 and the arrangement given in Table 5, respectively: GAVPI $36+$ NDSTC $33=69$ versus GREN 36 + VSDQ $33=69$; LKRQE $66+$ MFYWH $69=$ 135 versus THMFLP $(66-1)+$ KWCYAI $(69+1)=135$.]

In this analysis, it is important to recall that PAAS was generated based on the chemical similarity of AAs, with strict adherence to the two most important Mendeleevian principles: the continuity principle and the minimum change principle. When this is the case, then it makes sense, except through the golden mean (Rakočević, 1998, 2011), to test the order of AAs on the binary-code tree of GC (Figure 1) and from the neighborhood - non-neighborhood point of chemical pairs of AAs, starting from the simplest AA, glycine. We first find out the existence of neighborhood pairs, and members within pairs, in the alanine type, and the absence of a neighborhood in the simpler pair of non-alanine AAs $(\mathrm{G}-\mathrm{V})$; all together that are the regularities in the set of the first four pairs (Table 3). Also there is the absence of a neighborhood at the next alanine AAs and the neighborhood at a more complex pair of non-alanine AAs (P-I); all together that are the regularities in the set of the next six pairs of AAs (Table 4).

The test results, analogous to the results in Tables 1 and 2, are given in Tables 5 and 6 , in correspondence with Figures $2 \& 3$. Balancing and nuancing of the number of atoms in the side chains of AAs, correspondent to the physicochemical properties of amino acid molecules, are more than obvious.

\section{Box 1. Explanation of Figure 2}

"The Cyclic Invariant Periodic System (CIPS) of canonical AAs. At the [index] - the atom number within amino acid side chains. In the middle position there are chalcogene AAs (S, T \& $C, M$ ); follow - in next 'cycle' - the AAs of non-alanine stereochemical types (G, P \& V, I) [in red color], then two double acidic AAs with two their amide derivatives (D, E \& N, Q) [in blue color], the two original aliphatic AAs with two amine derivatives (A, L \& K, R) [in orange and yellow color]; and, finely, four aromatic AAs $(\mathrm{F}, \mathrm{Y} \& \mathrm{H}, \mathrm{W})$ - two up and two down. ... Notice that each amino acid position in this CIPS is strictly determined and none can be changed" (Rakočević, 2011, Fig. 6, p. 632). Note in this Supplement: In the previous paper (Rakočević, 2019a, Box 4) the CIPS (as here in Figure 2) was called CIPS I, because we introduced CIPS II there, and now we are introducing CIPS III in Figure 3.

\footnotetext{
${ }^{4}$ On the four stereochemical types of AAs see in (Popov, 1989; Rakočević and Jokić, 1996).

5 It should be noted that the quantities 97-107 in Table 1 are directly related to sequence 25-36-61, the determinant of both chemical and genetic code (Rakočević, 2019a, Table A2, p. 29).
} 
Unlike the cyclicity in CIPS I, where cyclicity is expressed through pairs of AAs, in CIPS III cyclicity is more complex. First, we note the simultaneous similarity and difference with CIPS I. Namely, in CIPS I, the outer cycle consists of aromatic AAs, unlike all other aliphatic ones. On the other hand, in CIPS III, the outer cycle consists of AAs of the non-alanine stereochemical type, unlike any other belong to the alanine stereochemical type. The interior of CIPS I consists of AAs, as described in the legend of Figure 2 (given here in Box 1). The interior of CIPS III (in Figure 3) is chemically more complex in the following sense: in the upper part, in the outer "ring" of four AAs with simpler nitrogen-oxygen functions, four AAs, with more complex nitrogen-oxygen functions are closed; in the lower part, however, there are four aromatic AAs in the outer "ring" and in the interior there are only two sulfur AAs, aliphatic but different from all other aliphatic [in their molecules except the same four atoms $(\mathrm{H}, \mathrm{C}, \mathrm{N}, \mathrm{O})$ they also have a fifth $(\mathrm{S})$ as a "hetero" atom].

\section{References}

Popov, E.M., 1989. Strukturnaya organizaciya belkov. Nauka, Moskva.

Rakočević, M.M., 1998a. The genetic code as a Golden mean determined system. Biosystems, 46, 283-291.

Rakočević, M.M., 2018a. The Cipher of the Genetic Code. BioSystems 171 (2018) 31-47

Rakočević, M. M., 2018b. Analogies of Genetic and Chemical Code. Polyhedron, 153, 292-298.

Rakočević, M. M., 2019a. Protein amino acids as a complete (periodic) system. Chemia Naissensis, Vol 2, Issue 1, pp. 1-43.

Rakočević, M. M., 2019b. Genetic code as an image of the mirror image. Part I, OSF Preprints, DOI 10.31219/osf.io/xht4c.

Rakočević, M.M., Jokić, A., 1996. Four stereochemical types of protein amino acids: synchronic determination with chemical characteristics, atom and nucleon number. J. Theor. Biol. 183, 345-349. 


\section{S u r ve y}

Survey 1. The mirroring of (double) first Boolean triangle and crossing with "Golden sequence" 0-1-0-1-0-1 from the six-bit binary tree ${ }^{6}$

$00-11-22 / 22-11-00 \rightarrow 00-11-22-11-00[0$ as $000 ; 1$ as $001 ; 2$ as 010$]$
$(00-11-22-11-00)+[3(010=2)]+[2(101=5)]=02-13-24-16-05$
$02-13-24-16-05 / 20-31-42-61-50$

\section{F i g u r e s}

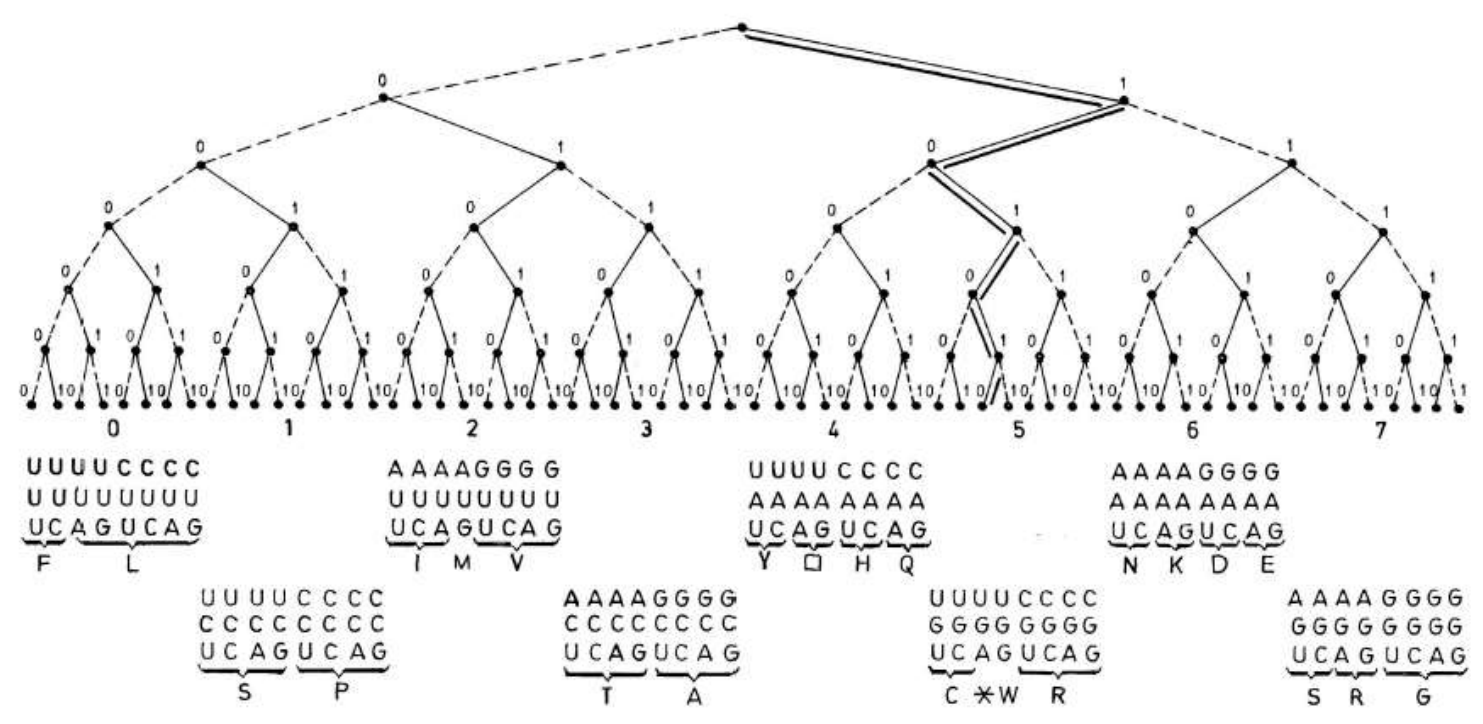

Figure 1. "Genetic code as a binary-code tree. The full lines: the routes of the greater (faster) changes from pyrimidine to purine or from two to three hydrogen bonds and vice versa. The dotted lines: the routes of the less (slower) changes. The double full line: the route of the maximum possible (fastest) changes; the route corresponding to the 'Golden mean route' on the Farey tree ... " (Rakočević, 1998, Fig. 1, p. 284).

\footnotetext{
6 The sequence 0-1-0-1-0-1 from the six-bit binary tree is a "Golden sequence" through the correspondence between the binary tree and Farey tree as we explained in a previous paper (Rakočević, 1998, Fig. 1 in relation to Fig. 2).
} 


\begin{tabular}{|cc|cc|}
\hline $\mathrm{F}_{14}$ & $\mathrm{Y}_{15}$ & $\mathrm{G}_{01}$ & $\mathrm{~V}_{10}$ \\
$\mathrm{~L}_{13}$ & $\underline{\mathrm{A}}_{04}$ & $\mathrm{R}_{17}$ & $\mathrm{~S}_{05}$ \\
$\mathrm{Q}_{11}$ & $\mathrm{~N}_{08}$ & $\mathrm{E}_{10}$ & $\mathrm{D}_{07}$ \\
$\mathrm{P}_{08}$ & $\mathrm{I}_{13}$ & $\mathrm{~N}_{08}$ & $\mathrm{Q}_{11}$ \\
$\mathrm{~T}_{08}$ & $\mathrm{M}_{11}$ & $\mathrm{~K}_{15}$ & $\mathrm{~T}_{08}$ \\
$\mathrm{~S}_{05}$ & $\mathrm{C}_{05}$ & $\mathrm{~W}_{18}$ & $\mathrm{H}_{11}$ \\
$\mathrm{G}_{01}$ & $\mathrm{~V}_{10}$ & $\mathrm{C}_{05}$ & $\mathrm{M}_{11}$ \\
$\mathrm{D}_{07}$ & $\mathrm{E}_{10}$ & $\mathrm{Y}_{15}$ & $\mathrm{~F}_{14}$ \\
$\mathrm{~K}_{15}$ & $\mathrm{R}_{17}$ & $\mathrm{~A}_{04}$ & $\mathrm{~L}_{13}$ \\
$\mathrm{H}_{11}$ & $\mathrm{~W}_{18}$ & $\mathrm{I}_{13}$ & $\mathrm{P}_{08}$ \\
Figure 2. CIPS I (Box 1) & Figure 3. CIPS III \\
\hline
\end{tabular}

\section{T a b l e s}

Table 1. The first four and following AAs in PAAS (Rakočević, 2019a)

\begin{tabular}{|c|c|c|c|c|}
\hline $\mathrm{G}_{01}$ & $\mathrm{~N}_{08}$ & & & \\
\hline $\mathrm{A}_{04}$ & $\mathrm{D}_{07}$ & 23 & 28 & $\rightarrow 51$ \\
\hline$V_{10}$ & $\mathrm{~S}_{05}$ & & & $(1 \times 51)$ \\
\hline $\mathrm{P}_{08}$ & $\mathrm{~T}_{08}$ & & Cross & $\rightarrow 107 / 97$ \\
\hline $\mathrm{I}_{13}$ & $\mathrm{C}_{05}$ & & & \\
\hline $\mathrm{L}_{13}$ & $M_{11}$ & & & \\
\hline $\mathrm{K}_{15}$ & $F_{14}$ & & & \\
\hline $\mathrm{R}_{17}$ & $Y_{15}$ & 79 & 74 & $\rightarrow 153$ \\
\hline $\mathrm{Q}_{11}$ & $W_{18}$ & $\downarrow$ & $\downarrow$ & $(3 \times 51)$ \\
\hline$E_{10}$ & $\mathrm{H}_{11}$ & 102 & 102 & \\
\hline
\end{tabular}


Table 2. The first five and following AAs in PAAS (Rakočević, 2019a)

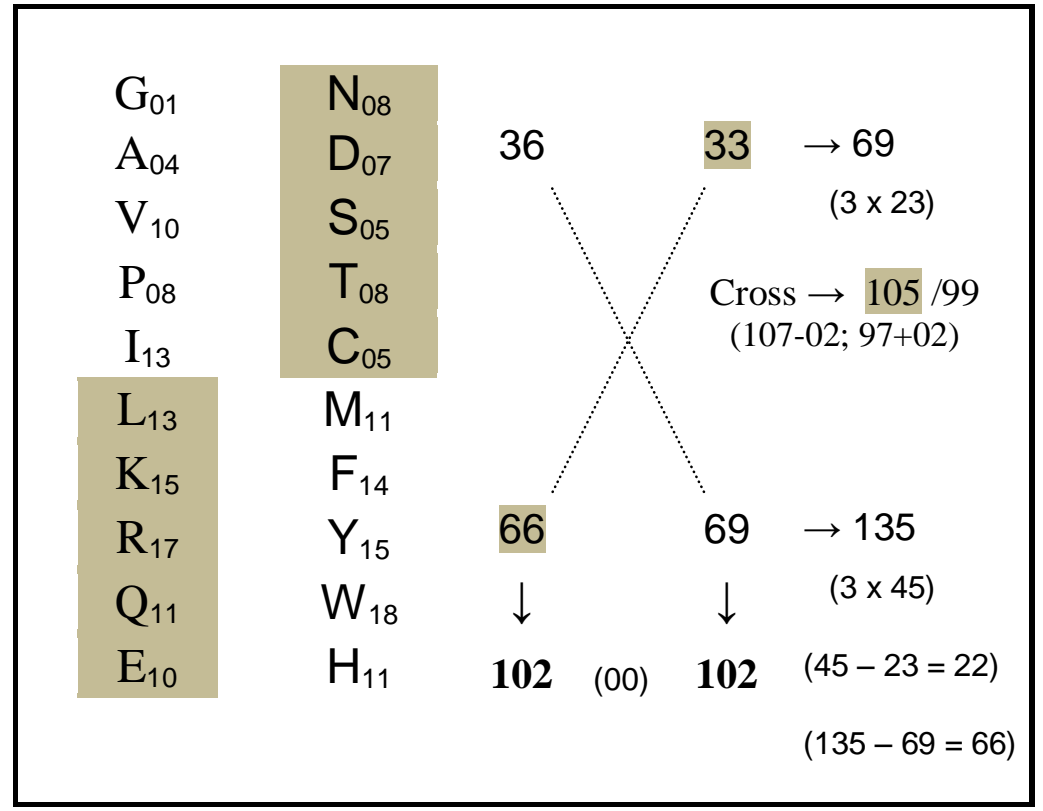

Table 3. Mostly adjacent AAs

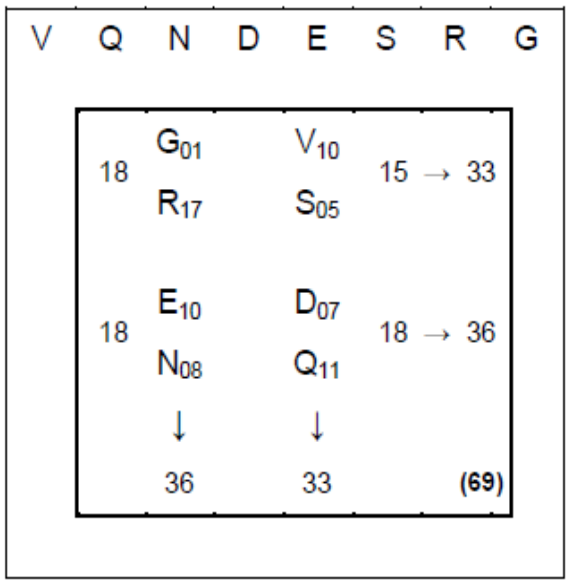

The adjacencies on Binary tree (Fig. 1)
Table 4. Mostly non-adjacent AAs

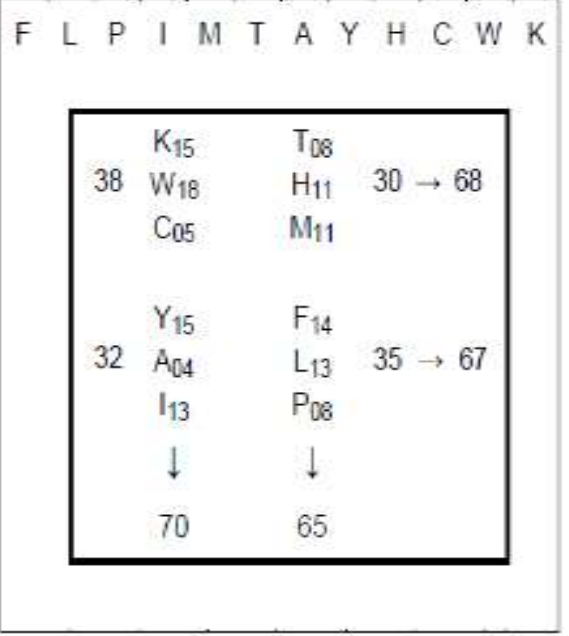


Table 5. The first four and following six AAs pairs

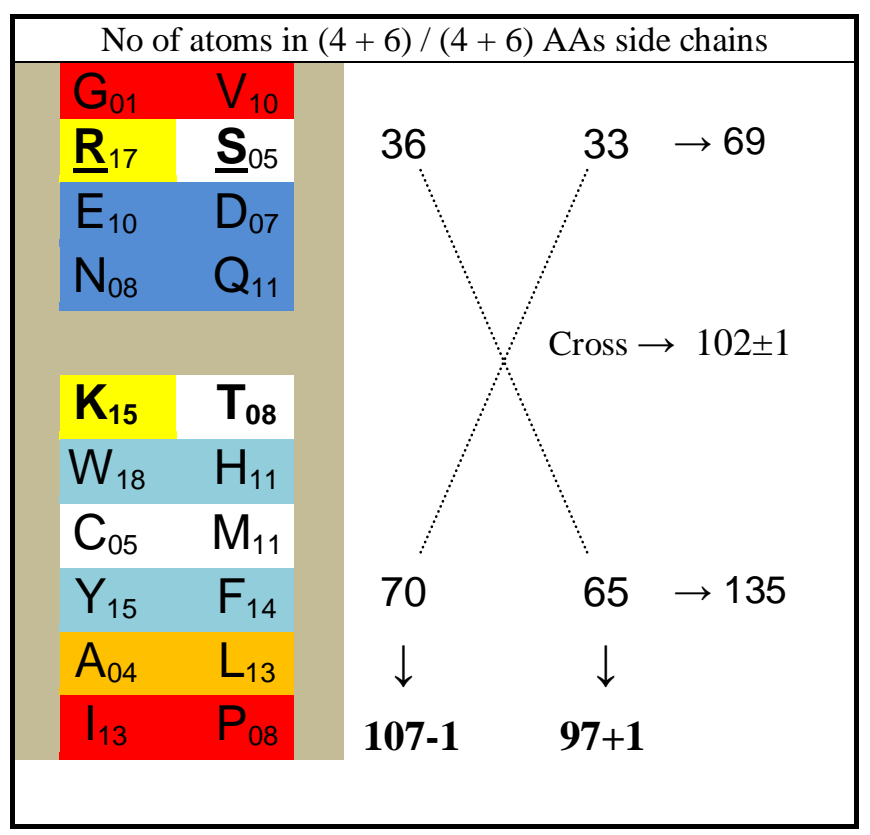

Table 6. The first five and following five AAs pairs

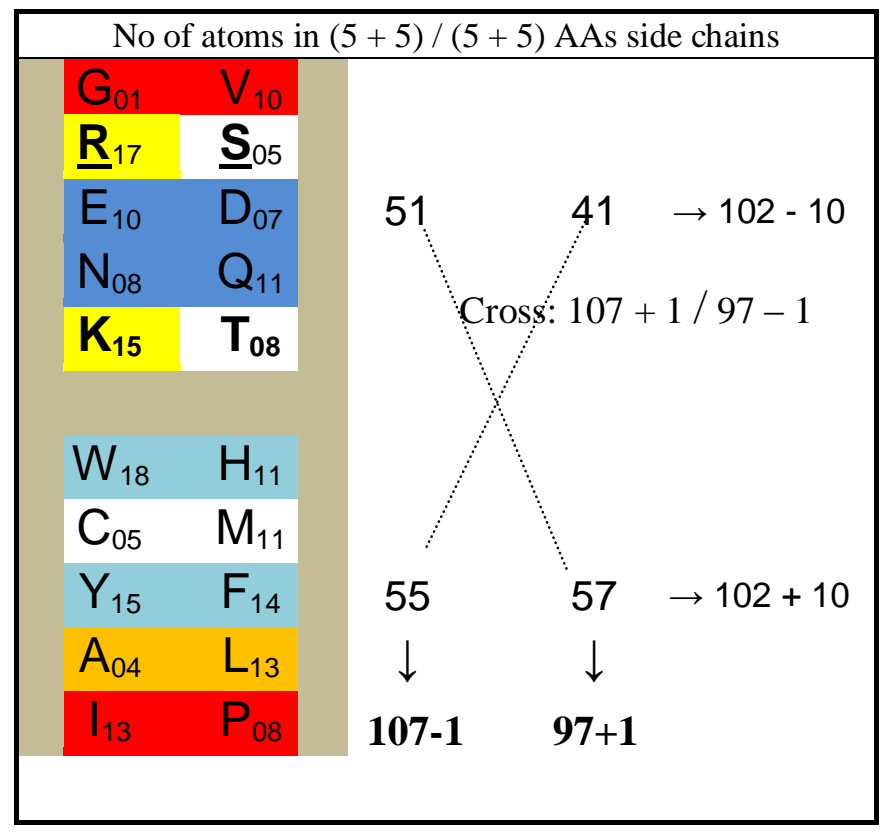




\title{
A note on the system chemistry
}

\author{
Miloje M. Rakočević \\ University of Niš, Faculty of Sciences and Mathematics, Department of Chemistry, \\ Višegradska 33, 18000 Niš, Serbia
}

\begin{abstract}
In one of our previous works (Rakočević, 2018b), we shown that there is an analogy of genetic and a chemical code. In this note however we would like to suggest that the reverse is true, the genetic code is a kind of natural systems analogous to the chemical system of chemical elements.
\end{abstract}

In one of our previous works (Rakočević, 2018b), we shown that there is an analogy of the genetic and the chemical code; in other words, the periodic system of chemical elements is a kind of natural codes. In this note, however, we would like to suggest that the reverse is true, the genetic code is a kind of natural systems analogous to the chemical system of chemical elements. The key definition of the periodic system of chemical elements is that there is a beginning and there is an end: the beginning, as the beginning of reativity; and the end, as an end to reactivity, is the appearance of inert elements.

[In another paper, we have also shown that it is necessary to distinguish between test tube chemistry and system chemistry (Rakočević, 2019b, Box 1). Now we show in what sense it is a system, when we say that a genetic code is a system. $\left.{ }^{2}\right]$

Figure 1 was originally given in (Rakočević, 2011, Figure 10, p. 835). That what is new here is a different shading and coloring, according to the new classification. Looking at Figure 1, we are trying to discover how here, in the system of genetic code, the beginning and the end of the system are realized. As we see the beginning and ending refer to native amino acids. [For further consideration, it should be recalled that Figure 1 also follows from the periodic system of numbers, PSN (Figure 2) in that the number of atoms per row is such that it is the same as the diagonal of the periodic system.]

Whether or not an amino acid is native is determined by comparison with the key chemical function in the "head" of the amino acid, which is the carboxyl group. We see that these are the only two amino acids, aspartic and glutamic, they represent only the similarity and self-similarity to the amino acid head, they are the beginning. From the

\footnotetext{
${ }^{1}$ The test tube chemistry means a chemical reaction in any vessel, from the first human hearth, through the retorts of alchemists to modern chemical vessels in chemical laboratories and/or in technological vessels and autoclaves.

${ }^{2}$ Already in the first paper we published in an international journal, we presented an important systemic characteristic of the genetic code - a synchronous determination with chemical characteristics and the number of atoms and nucleons (Rakočević and Jokić, 1996).
} 
standpoint of self-similarity, the path to the end should be such that it includes one more functional group from the "head", which is the only remaining one - the amino group. Two amide amino acids are obtained, and they represent the end; a line from amino acids with a carboxylic group in the side chain to amino acids with an amide group in the side chain was realized.

The native state in the amino acid molecule, apart from the main functional group, is certainly the amino acid skeleton, ie. the skeleton of the "head". The skeleton of the simplest hydrocarbon, methane, is meant. This pattern is repeated with the first amino acid of the alanine stereochemical type, with alanine; with its body being only one methyl group. Hence, alanine is a native amino acid. It also represents a "beginning".

The question is which amino acid is at the end? It certainly cannot be serine, but its analogue systeine, because it is the farthest from the starting point, possesses sulfur that is present neither in the first nor in the second period but in the third. (It is the only chemical element out of five that builds twenty protein amino acids, which is in the third period and the remaining four elements are in the first and second.)

In Figure 1, the native amino acids are represented in red. Following are three other native amino acids, these are aromatic amino acids - all but one, tyrosine. Because phenylalanine has a $\mathrm{CH}_{2}$ group between head and body, it belongs to the alanine stereochemical type, in other words it has a structural pattern of a methyl group, that is, a methane molecule. Starting from it there are two ends, one is with tryptophan where besides benzene there is also a pyrrole ring; but the amino acid histidine also has an end function because it also has a six pi electron system as we explained in the paper (Rakočević, 2018a)

Three (LSR) plus four (TM KY) non-native amino acids remain, which we have referred to as "intruders" in previous work (Rakočević, 2019b, Section 2.4) and explained their uniqueness. Finally, there are four amino acids of the non-alanine stereochemical type in the far right column. tipa. ${ }^{3}$

U Tabelama 1 i 2 pokazano na koji je jedinstven način klasifikacija of Aas in Figure 1 praćene ravnotežom broja nukleona u klasama i podklasama, a sve u relaciji sa unikatnostima periodnog sistema brojeva u decimalnom brojevnom sistemu, prikazanom na slici 2. Svi rezultati, zajedno, idu u priloh našoj hipotezi da je genetski kod bio kompletan još preb iotski, i kao takav, bio je uslov za nastanak života.

Tables 1 and 2 show in which a unique way the classification of AAs in Figure 1 followed by the balance of number of nucleons in classes and subclasses; all in relation to

\footnotetext{
${ }^{3}$ For four stereochemical types of protein amino acids, see: (Popov, 1989) and (Rakočević, Jokic, 1996).
} 
the uniqueness of the periodic number system in the decimal number system, shown in Figure 2. All results, together, they support our hypothesis that the genetic code was complete yet prebiotic, and as such, was a condition for the origin of life (Rakočević, 2004).

\section{References}

Popov, E.M., 1989. Strukturnaya organizaciya belkov. Nauka, Moskva.

Rakočević, M.M., 1998a. The genetic code as a Golden mean determined system. Biosystems, 46, 283-291.

Rakočević, M.M., 2004. A harmonic structure of the genetic code. J. Theor. Biol. 229, 221-234.

Rakočević, M.M., 2011b. Genetic code as a coherent system. Neuroquantology 9 (4), 821-841.

Rakočević, M.M., 2018a. The Cipher of the Genetic Code. BioSystems 171 (2018) 31-47

Rakočević, M. M., 2018b. Analogies of Genetic and Chemical Code. Polyhedron, 153, 292-298.

Rakočević, M. M., 2019a. Protein amino acids as a complete (periodic) system. Chemia Naissensis, Vol 2, Issue 1, pp. 1-43.

Rakočević, M. M., 2019b. Genetic code as an image of the mirror image. Part I, OSF Preprints, DOI 10.31219/osf.io/xht4c.

Rakočević, M. M., 2019c. Genetic code as an image of the mirror image. Supplement 1, OSF Preprints, DOI 10.31219/osf.io/umnt3

Rakočević, M.M., Jokić, A., 1996. Four stereochemical types of protein amino acids: synchronic determination with chemical characteristics, atom and nucleon number. J. Theor. Biol. 183, 345-349. 


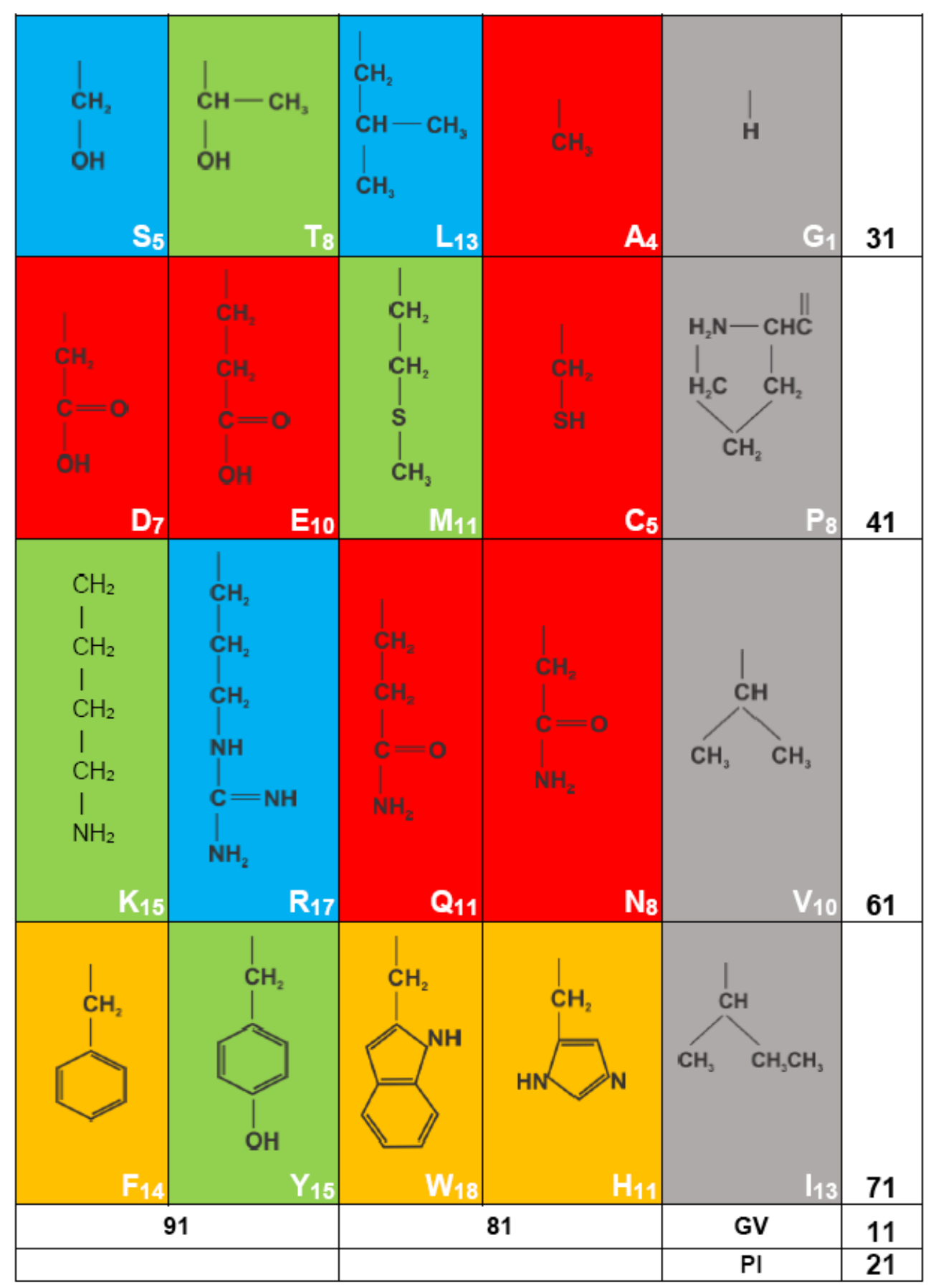

Figure 1. The chemical system of the Genetic code: $6+3$ native AAs, $3+4$ non-native AAs, altogether 16 AAs of alanine stereo-chemical type plus 4 AAs of non-alanine stereo-chemical type. Natives: red plus orange; non-natives ("intruders") blue plus green AAs and four AAs as a second class with gray color. 


\begin{tabular}{|c|c|c|c|c|c|c|c|c|c|c|c|}
\hline &.. & & & & & & & & & & \\
\hline & -21 & -20 & -19 & -18 & -17 & -16 & -15 & -14 & -13 & -12 & -11 \\
\hline & -10 & -09 & -08 & -07 & -06 & -05 & -04 & -03 & -02 & -01 & 00 \\
\hline & 01 & 02 & 03 & 04 & 05 & 06 & 07 & 08 & 09 & 10 & 11 \\
\hline (2) & 12 & 13 & 14 & 10 & $16^{-}$ & $-47^{--}$ & -18 & 19 & 20 & 21 & 22 \\
\hline (3) & 23 & 24 & 25 & $26^{\prime}$ & 27 & 28 & 29 & 30 & 31 & 32 & 33 \\
\hline (4) & 34 & 35 & 36 & 37 & 38 & 39 & 40 & 41 & 42 & 43 & 44 \\
\hline (5) & 45 & 46 & 47 & 48 & 49 & 50 & $\underline{51}$ & 52 & 53 & 54 & 55 \\
\hline (6) & 56 & 57 & 58 & 59 & 60 & 61 & 62 & 63 & 64 & 65 & 66 \\
\hline (7) & 67 & 68 & 69 & 70 & 71 & 72 & 73 & 74 & 75 & 76 & 77 \\
\hline (8) & 78 & 79 & 80 & 81 & 82 & 83 & 84 & 85 & 86 & 87 & 88 \\
\hline (9) & 89 & 90 & 91 & 92 & 93 & 94 & 95 & 96 & 97 & 98 & 99 \\
\hline (A) & $\overline{A O}$ & $\mathrm{~A} 1$ & A2 & $\mathrm{A3}$ & $\overline{A 4}$ & $\overline{A 5}$ & $\overline{A 6}$ & A7 & A8 & A9 & AA \\
\hline (B) & B1 & B2 & B3 & B4 & B5 & B6 & B7 & B8 & B9 & $B A$ & $\mathrm{BB}$ \\
\hline
\end{tabular}

Figure 2. Periodic system of the numbers taken from (Rakočević, 2011, Tab 4, p. 826) and (Rakočević, 2019a, Fig. A1, p. 28). 
Table 1. Distinctions of AAs through chemical properties and number of nucleons in distinctive classes

Green KYTM $299+$ Blue LSR $188=\mathbf{4 8 7}$

$\operatorname{Red}$ AC $62+[$ DE $132+$ NQ $130=262]=\mathbf{3 2 4}=18 \times 18$

Orange FWH $302(302+324=\mathbf{6 2 6}[\mathbf{6 2 6}+\mathbf{4 8 7}=1111+2$

Green KYTM 299 - Blue LSR $188=111$
$\operatorname{Red}(\mathrm{AC}$ DE NQ) 324 - Orange (FWH) $302=22$

Table 2. Relations of the first two numbers with the first possible (double) Boolean space triangle in PSN by generating a series of odd and even numbers (as sets of units)

$$
\begin{aligned}
& (1+22) \&(11+2) \rightarrow 23>13 \\
& 110 / 1100 \\
& (111+22) \&(1111+2) \rightarrow 133<1113 \\
& 11000 \text { / } 110000 \\
& (11111+22) \&(111111+2) \rightarrow 11133<111113 \\
& 1100000 \text { / } 11000000 \\
& (1111111+22) \&(11111111)+2 \rightarrow 1111133<11111113 \\
& \text {....... }
\end{aligned}
$$




\title{
Genetic code as an image of the mirror image. Supplement 2
}

\author{
Miloje M. Rakočević \\ University of Niš, Faculty of Sciences and Mathematics, Department of Chemistry, \\ Višegradska 33, 18000 Niš, Serbia
}

\begin{abstract}
In this (second) Supplement are given the further new insights on mirror symmetry within Genetic Code (GC). The main result is the correspondence of the binary tree of GC and the Standard GC Table in such a way that it follows that it makes no sense to talk about the evolution of GC from the aspect of test tube chemistry (of performing chemical reactions in nature and/or laboratory), but only makes sense to talk about prebiotic chemical evolution of GC in a manner analogous to the evolution of the chemical code i.e. Periodic system of chemical elements. Taken together, an even more important conclusion is that all the physical and chemical laws we know represent immediate causality, while in the case of GC on the scene is an indirect causality, realized through multiple mirror symmetry.
\end{abstract}

Keywords: Protein amino acids, Genetic code, Binary tree, Golden mean, Periodic system, Mirror image. Mirroring.

In Supplement 1 of this paper, we outlined the arrangement of amino acid pairs, in the order from the last to the first amino acid (AA) in the GC binary tree (Suppl. 1, Fig. 3 in relation to Fig. 1) ${ }^{1}$. In doing so, the first member of the pair was selected with strict adherence to two Mendeleevian principles - the principle of minimum change and the principle of continuity. [The only exception is the choice of asparagine rather than lysine because asparagine is a pair-member of aspartic acid, despite aspartic acid being previously paired with glutamic acid.]

In the meantime, we have presented the distinction of amino acid molecules by the formula: $[(6+3)+(3+4)+4]]^{2}$ (Rakočević, 2020a), where subset $\{6+3\}$ represents native AAs, subset $\{3+4\}$ no-native AAs; both subsets within the set of $\{16\}$ AAs of the alanine stereochemical type. The last number in summation is a set of $\{4\}$ AAs of non-alanine stereochemical types. ${ }^{3}$ Now it makes sense to combine the two distinctions (the order of

\footnotetext{
${ }^{1}$ Figure 3 in Suppl. 1, here is Figure 1. Table 1 and Table 2 here contain the distribution of AAs in Table of standard GC. Table 3 shows, among other things, the pairs of AAs by chemical similarity. In relation to the pairing in Figure 1, here is the original chemical pairing: S-T and K-R but in Figure 1 the pairing is S-R and T-K. And, there is another pairing: here, in Table 3, the pairs are G-G (because glycine is the only one AA in the glycine stereochemical type; P-P (because proline is the only one in the proline type), V-I (because they are the only two AAs in the valine stereochemical type). In Figure 1, the pairs are G-V and P-I, as in CIPS 2 (Rakočević, 2019a; Fig. 1, p. 6).

${ }^{2}$ It should be noted that number 4 is a neighbor, i.e. a follower of number 3 in the series of natural numbers (which is a condition for the validity of two Mendelian principles). We will hereafter refer to this formula as the Kinship Formula. Our prediction is that the Kinship Formula is a unique situation in a specific arithmetic system, which we will address in the next paper (Rakočević, 2020b).
}

${ }^{3}$ On the four stereochemical types of AAs see in (Popov, 1989; Rakočević and Jokić, 1996). 
the amino acid pairs on the binary tree and the in-out arrangement in Table of GC), and look at how they correspond to each other. This relationship is shown to be reality, so here we present it in Figure 1 in relation to Tables 1 and 2.

Figure 1 is the same as that presented in Suppl. 1 as Fig. 3, with new distinctions given here; there were differences in relation to the golden mean of the binary tree of GC, and here are the distinctions to the chemical affinity of amino acids as constituents of GC

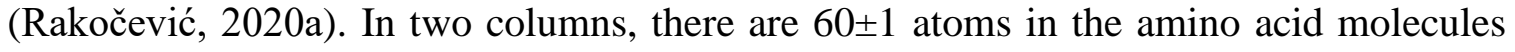
(in their side chains) at odd positions of the total number of atoms of distinct amino acid classes (designated by different colors), respectively. [(14+27+18 = 59); $(18+18+25=$ 61).] In even positions: 47 and 37, respectively. $[(17+30=47) ;(18+19=37) .]^{4}$ (Box 1$)$

\section{Box 1. Explanation of Figure 1}

This Figure corresponds to Figure 3 in Suppl. 1. All pairs of AAs within alanine stereochemical type correspond to pairs in the arrangement given in Table 3, except in two cases. The positions of AAs on the binary tree are such that the restructuring of S-T and K-R pairs into S-R and T-K is inevitable. The generation of two pairs within non-alanine stereochemical types is explained in footnote 1 .

And the total generation of the pairs is as follows: Going from right to the left, from end towards the beginning of the binary tree of GC (Fig. 1 in Suppl. 1) we first encounter glycine (G), and then look for its pair-member, amino acid valine $(\mathrm{V})$; then, in the same way we find R-S and then D-E; we skip K, and take derivatives of pair D-E, which is pair N-Q. Only then do we return to skipped lysine $(\mathrm{K})$ and, going forward, look for its new pair-member, threonine $(\mathrm{T})$. After selecting lysine $(\mathrm{K})$, the first following AA is tryptophan (W). We take it and go looking for its pair-member histidine $(\mathrm{H})$. Then comes cysteine $(\mathrm{C})$, whose pair-member methionine $(\mathrm{M})$ is located far from there. The following is tyrosine $(\mathrm{Y})$ whose pair-member phenylalanine $(\mathrm{F})$, exists at the very beginning of the binary tree.

The next unselected AA is alanine (A), which we take as the next, along with its pair-member leucine (L). Finally, there is isoleucine (I), in the immediate vicinity of which is its pair-member, proline $(\mathrm{P})$.

Six native aliphatic AAs are marked in red; orange: three native aromatic; follow $3+4$ non-native AAs (blue plus green, respectively). Altogether: 16 AAs of alanine stereochemical type, in color; plus 4 AAs of non-alanine stereochemical type, without color.

Notice also direct and cross balances within columns: $(51+55=107-1) ;(41+57=97+1) ;(51+57$ $=107+1) ;(41+55=97-1)$. These relations correspond to the arithmetic system presented in: (Rakočević, 2019a, Tab. A2, p. 29);

\footnotetext{
${ }^{4}$ That the principles of nuancing and balancing are valid here is more than obvious (footnote 2 in Suppl. 1).
} 
The arithmetic form of the results that came from the linear order of AAs on a binary tree is analogous to the result obtained in chemically relevant distinctions of amino acid molecules in the Standard GC Table, realized not in linear but in inner-outer arrangement (Tables 1 and 2 in relation to Survey 1).

In Survey 1 it is shown that the positions of AAs on the binary tree of GC and in the Standard GC Table are interconnected by multiple mirror symmetries, which symmetries are expressed over the notations of the number of atoms in the side chains of amino acid molecules.

From the presented results it follows that there is no point in talking about the evolution of GC from the aspect of test tube chemistry (of performing chemical reactions in nature and / or in the laboratory), but only makes sense to talk about system chemistry, that means to talk about prebiotic chemical evolution of GC in a manner analogous to the evolution of the chemical code i.e. Periodic system of chemical elements. ${ }^{5}$ Taken together, an even more important conclusion is that all the physical and chemical laws we know represent immediate causality, while in the case of GC on the scene is an indirect causality, realized through multiple mirror symmetry. ${ }^{6}$

Everything presented goes in support of the conclusion of the original paper of which this is the Supplement. First, in support of the working hypothesis contained in the title of the article, and also in support of the hypothesis of the analogy of GC with quantum physics, and the hypothesis that GC was still prebiotic complete.

\footnotetext{
5 See the original paper of this Supplement for the test tube chemistry and system chemistry (Rakočević, 2019b, Box 1).

${ }^{6}$ When it comes to mirror symmetry within the binary number system, which we have dealt with in the three works mentioned in 2019, then we have presented not only direct mirroring according to the notations [(010/010) (101/101)], but also indirect. by the opposite of the digits, analogous to Dirac's mirror symmetry positron / electron, in the following sense: [(010/101) (101/010)] (Rakočević, 2019b, footnote 9).
} 


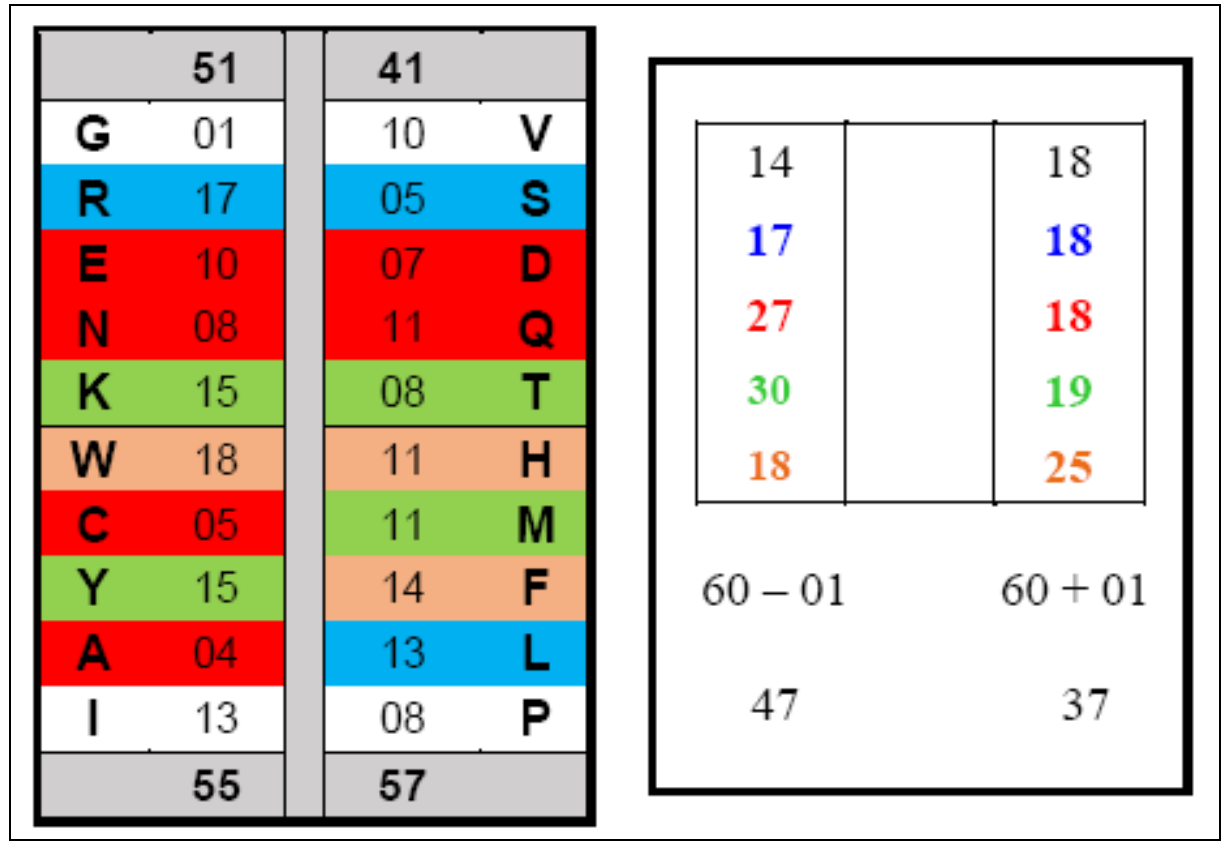

Figure 1. The Pairs of AAs taken from the binary tree of GC, going from right to left, i.e. from end to beginning. In selecting the first member, in the mating process, two Mendeleevian principles, the minimum change principle and the continuity principle, are respected. The second member of the pair automatically joins (from close proximity or, for a leap, from distance), wherever it is on a binary tree. The same rule applies to the classes of AAs in this Figure. For example, the number of atoms in the amino acids (in their side chains) located in the first red region are automatically joined, in the summation process, by the number of atoms from all the following red areas, as shown on the right side of the Figure. [We used the same procedure to read and take 7 "golden" AAs and their 7 complements, as well as 6 non-complements, on the binary tree of GC (Rakočević, 1998, 2011). A, also the same procedure for reading 20 AAs in the Standard GC Table, when equilibrium determination of AA classes was shown in even seven parameters (Rakočević, 2004, Tables 7 and 8, p. 228.] 
Table 1. Amino acid positions in standard GC (I)

\begin{tabular}{|c|c|c|c|}
\hline $\begin{array}{l}\mathrm{F}_{14} \\
\mathrm{~L}_{13}\end{array}$ & $\mathrm{~S}_{05}$ & $Y_{15}$ & $\begin{array}{l}\mathrm{C}_{05} \\
\mathrm{~W}_{18}\end{array}$ \\
\hline$L_{13}$ & $\mathrm{P}_{08}$ & $\begin{array}{l}\mathrm{H}_{11} \\
\mathrm{Q}_{11}\end{array}$ & $\mathrm{R}_{17}$ \\
\hline $\begin{array}{c}\mathrm{I}_{13} \\
\mathrm{M}_{11}\end{array}$ & $\mathrm{~T}_{08}$ & $\begin{array}{l}N_{08} \\
K_{15}\end{array}$ & $\begin{array}{l}S_{05} \\
R_{17}\end{array}$ \\
\hline$V_{10}$ & $A_{04}$ & $\begin{array}{l}\mathrm{D}_{07} \\
\mathrm{E}_{10}\end{array}$ & $\mathrm{G}_{01}$ \\
\hline \multicolumn{4}{|c|}{$\begin{array}{c}61 / 61[59,61] \\
61-59=02 \\
61-61=00 \\
122-120=02\end{array}$} \\
\hline
\end{tabular}

All AAs in outer (red) area are nonpolar measured by cloister energy; in inner (blue) area polar are all, except one: histidine is ambivalent in cloister energy, but polar in hydropathy. In amino acids (within their side chains) at outer/inner areas there are 61/61 atoms, respectively. Polar/nonpolar squares: $4 \pm 0$. (The polarity/nonpolarity data in Table 3.) [The Table represents the reduced form of Table 3 in (Rakočević, 2017).]

Table 2. Amino acid positions in standard GC (II)

\begin{tabular}{|c|c|c|c|}
\hline $\begin{array}{l}F_{14} \\
L_{13}\end{array}$ & $\mathrm{~S}_{05}$ & $Y_{15}$ & $\begin{array}{l}\mathrm{C}_{05} \\
\mathrm{~W}_{18}\end{array}$ \\
\hline $\mathrm{L}_{13}$ & $P_{08}$ & $\begin{array}{l}\mathrm{H}_{11} \\
\mathrm{Q}_{11}\end{array}$ & $\mathrm{R}_{17}$ \\
\hline $\begin{array}{l}\mathrm{I}_{13} \\
\mathrm{M}_{11}\end{array}$ & $\mathrm{~T}_{08}$ & $\begin{array}{l}\mathrm{N}_{08} \\
\mathrm{~K}_{15}\end{array}$ & $\begin{array}{l}\mathrm{S}_{05} \\
\mathrm{R}_{17}\end{array}$ \\
\hline $\mathrm{V}_{10}$ & $\mathrm{~A}_{04}$ & $\begin{array}{l}D_{07} \\
E_{10}\end{array}$ & $\mathrm{G}_{01}$ \\
\hline \multicolumn{2}{|c|}{$\begin{array}{l}59 / 58[59,61] \\
59-47=\mathbf{1 2} / 21 \\
58-37=\mathbf{2 1} / 12\end{array}$} & \multicolumn{2}{|c|}{$\begin{array}{l}59-37=22 \\
58-47=11\end{array}$} \\
\hline
\end{tabular}

All AAs in right (pink) area plus dark-green are polar, measured by cloister energy and hydropathy. On the left in light-green area are nonpolar. Polar/nonpolar squares: $4 \pm 1$. In amino acids (within their side chains), in right/left areas there are 59/58 atoms, respectively. [The Table represents the reduced form of Table 4 in (Rakočević, 2017).] 
Table 3. Pairing process between 16 AAs of alanine stereochemical type and two times 4 AAs od non-alanine stereochemical types

$\left.\begin{array}{ccccc}\hline \mathrm{CE} & \mathrm{HP} & \mathrm{I} & \\ -0.16 & -0.4 & \mathrm{G} & 01 \\ -0.09 & +1.8 & \mathrm{~A} & 04 \\ -0.52 & +4.2 & \mathrm{~V} & 10\end{array}\right]$ (12)

After (Rakočević and Jokić, 1996, p. 346): On the full line, as well as on the dotted line, there are 118 atoms; HP: hydropathy index on a number unnamed scale (Kyte and Doolittle, 1982); CE: cloister energy in $\mathrm{kcal} / \mathrm{moll}$ (Swanson, 1984). 
Survey 1. Arithmetic relations of positions of AAs on the binary tree and in the Standard GC Table

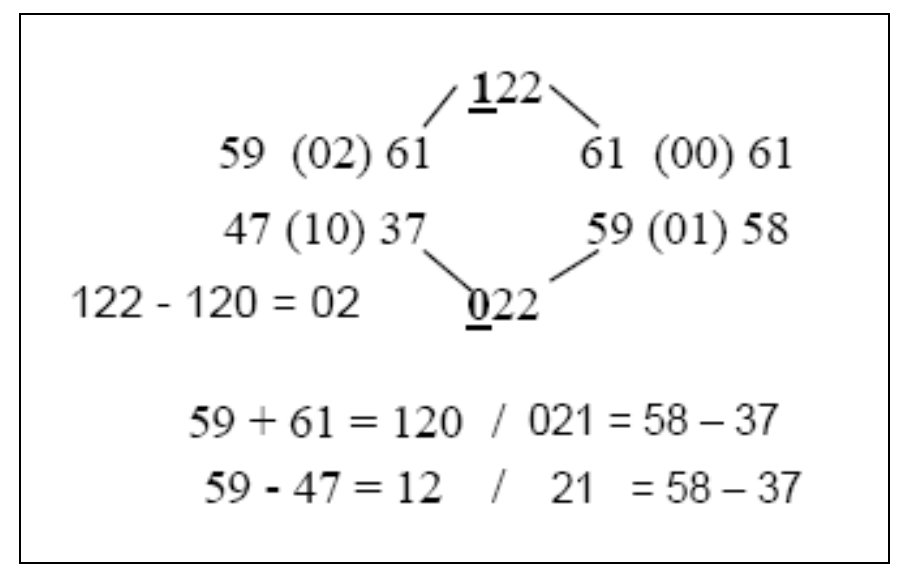

Above left are the sums of the number of atoms $(59,61 ; 47,37)$ in AAs on the binary tree of GC, calculated according to Figure 1 ; on the right $(61,61 ; 59,58)$ according to Tables 1 and 2 , respectively. Mirror symmetries ("hidden") appear through the addition and/or subtraction operations as visible patterns. 


\section{References}

Kyte, J., Doolittle, R.F., 1982. A simple method for displaying the hydropathic character of a protein. J. Mol. Biol. 157, 105-132.

Popov, E.M., 1989. Strukturnaya organizaciya belkov. Nauka, Moskva.

Rakočević, M.M., 1998a. The genetic code as a Golden mean determined system. Biosystems, 46, 283-291.

Rakočević, M.M., 2004. A harmonic structure of the genetic code. J. Theor. Biol. 229, 221-234.

Rakočević, M.M., 2011. Genetic code as a coherent system. Neuroquantology 9 (4), 821-841.

Rakočević, M.M., 2017. Golden and Harmonic Mean in the Genetic code. OSF Preprints DOI 10.31219/osf.io/2pfe7.

Rakočević, M.M., 2018a. The Cipher of the Genetic Code. BioSystems 171, 31-47.

Rakočević, M. M., 2018b. Analogies of Genetic and Chemical Code. Polyhedron, 153, 292-298.

Rakočević, M. M., 2019a. Protein amino acids as a complete (periodic) system. Chemia Naissensis, Vol 2, Issue 1, pp. 1-43.

Rakočević, M. M., 2019b. Genetic code as an image of the mirror image. Part I, OSF Preprints, DOI 10.31219/osf.io/xht4c.

Rakočević, M. M., 2019c. Genetic code as an image of the mirror image. Supplement 1, OSF Preprints, DOI 10.31219/osf.io/umnt3

Rakočević, M. M., 2020a. A note on the system chemistry, OSF Preprints, DOI 10.31219/osf.io/z7bm3.

Rakočević, M. M., 2020b. A note on the system chemistry (II). In preparation.

Rakočević, M.M., Jokić, A., 1996. Four stereochemical types of protein amino acids: synchronic determination with chemical characteristics, atom and nucleon number. J. Theor. Biol. 183, 345-349.

Swanson, R., 1984. A unifying concept for the amino acid code. Bull. Math. Biol. 46, 187-207. 


\title{
Appendix
}

\section{Genetic code as an image of the mirror image. Part I}

\author{
Epilogue
}

Ms. No. xxxxxxxxx

Title: Genetic code as an image of the mirror image. Part I Corresponding Author: Professor Miloje Rakocevic

Dear Professor Rakocevic,

We regret to inform you that your manuscript has not been deemed acceptable for publication in the Journal xxxxxxxxxxxx. Below please find the reviewer evaluation(s).

As our journal's acceptance rate averages less than half of the manuscripts submitted, regretfully, many otherwise good papers cannot be published by Journal xxxxxx.

Sincerely,

Professor xxxxxxxxx

Chief Editor

Journal $x x x x x x x x x x x x x x$

Reviewers' comments:

Reviewer \#5: The manuscript submitted by Miloje M. Rakočević described facts that support the hypothesis that genetic code is a specific image of a specific mirror image. The author also claimed that these facts align with quantum physics. This work is well supported by the evidences provided by the author. The work describes the origin of genetic code and it is prebiotic complete. I found this work address a scientific question that is an old but quite important. However the author described the work in a very difficult angle for the general audience to understand. Thus it loses the general interests of this work. I support its publication if the author can introduce or redescribe the work in common language. This work is too mathematical for general biology readers to understand.

Minor issue: The current development of unnatural amino acids and fifth genetic code other than ATCG may arise new question for the study in this work. Does the author have any comment on these expanded genetic codes? 